\title{
Prevalence of lymph node metastases in superficial esophageal squamous cell carcinoma
}

\author{
Bin Li, MD, ${ }^{\text {a,b }}$ Haiquan Chen, MD, ${ }^{\text {a,b }}$ Jiaqing Xiang, MD, ${ }^{\text {a,b }}$ Yawei Zhang, MD, ${ }^{\text {a,b }}$ Yunyi Kong, MD, ${ }^{\text {b,c }}$ \\ David H. Garfield, MD, ${ }^{\mathrm{d}}$ and Hecheng Li, MD ${ }^{\mathrm{a}, \mathrm{b}}$
}

Objective: Endoscopic treatment of superficial esophageal carcinoma has been increasingly conducted around the world. Because no lymph nodes are removed in such a procedure, the risk of lymph node metastases (LNMs) should be clearly understood. The aim of the present study was to accurately clarify the pattern of lymphatic spread in patients with superficial esophageal squamous cell carcinoma and analyze the factors potentially related to LNMs.

\begin{abstract}
Methods: The pattern of lymphatic spread was studied in 189 patients who had undergone radical lymphadenectomy from 2006 to 2011. The risk factors associated with LNMs were determined by multivariate logistic regression analysis. According to the depth of tumor invasion, mucosal tumors were classified as M1, M2, and M3 and submucosal tumors as SM1, SM2, and SM3.

Results: A total of 4252 lymph nodes were resected (average, $23 \pm 9$; range, 12-68). LNMs occurred in 49 patients $(25.9 \%)$. The frequency of LNMs was $4.3 \%$ in those with mucosal and $33.1 \%$ in those with submucosal cancer. LNMs were found in $0 \%, 0 \%, 11.8 \%, 24.0 \%, 20.5 \%$, and $43.8 \%$ of the M1, M2, M3, SM1, SM2, and SM3 cancer, respectively. For submucosal cancer, SM3 cancer $(P=.006)$ and lymphovascular invasion $(P=.001)$ were significant independent risk factors for LNMs. Paratracheal nodes were the most frequently involved. "Skip" metastases occurred in 20 of 49 patients $(40.8 \%)$.
\end{abstract}

Conclusions: Endoscopic treatment can be attempted when the tumor is limited to the lamina propria mucosa. However, 2-field radical lymphadenectomy with careful upper mediastinal lymph node resection should be conducted for submucosal squamous cell carcinoma. (J Thorac Cardiovasc Surg 2013;146:1198-203)

Earn CME credits at

http://cme.ctsnetjournals.org

Superficial esophageal cancer (SEC), defined as intraepithelial (Tis), mucosal (T1a), and submucosal (T1b) cancer, regardless of lymph node status, implies a better prognosis owing to an early tumor stage. ${ }^{1}$ Traditionally, esophagectomy with radical lymphadenectomy has been considered the best curative treatment for such patients. However, this procedure carries significant long-term morbidity. ${ }^{2-4}$ To achieve a better postoperative quality of life, a trend has occurred toward endoscopic treatment of superficial lesions. ${ }^{5,6}$ Nonetheless, the potential for lymph node metastases (LNMs)

From the Department of Thoracic Surgery ${ }^{\mathrm{a}}$ and Department of Pathology, ${ }^{\mathrm{c}}$ Fudan University Shanghai Cancer Center, Shangai, China; Department of Oncology, Shanghai Medical College, Fudan University, Shangai, China; and ProMed Cancer Centers, ${ }^{\mathrm{d}}$ Shanghai, China.

Disclosures: Authors have nothing to disclose with regard to commercial support.

Received for publication Feb 22, 2013; revisions received April 26, 2013; accepted for publication July 9, 2013; available ahead of print Aug 28, 2013.

Address for reprints: Haiquan Chen, MD, Department of Thoracic Surgery, Fudan

University Shanghai Cancer Center, No. 270 Dong'an Rd, Shanghai 20032, China

(E-mail: hqchen1@yahoo.com).

$0022-5223 / \$ 36.00$

Copyright (๑) 2013 by The American Association for Thoracic Surgery

http://dx.doi.org/10.1016/j.jtcvs.2013.07.006 exists, because of the abundant lymph-capillary plexus in the lamina propria mucosa and submucosa of the esophagus. At present, treatment of SEC remains somewhat controversial, including the most appropriate criteria for endoscopic therapy, patient selection for esophagectomy, and extent of lymphadenectomy., ${ }^{3,7-10}$ Because no lymph nodes are removed during endoscopic therapy and radical lymphadenectomy to remove all potentially involved nodes is thought to be essential for curative treatment, it is important to assess the likelihood of LNMs in the management of the disease.

The present study represents the first series of SEC from China, where squamous cell carcinoma (SCC) is the predominant pathologic type. The aim of the present retrospective study was to accurately document the pattern of LNMs in superficial SCC and to study the potential associated factors.

\section{METHODS \\ Patients}

The institutional review board of Fudan University Shanghai Cancer Center approved the present retrospective study. From January 2006 to December 2011, 2069 patients underwent esophagectomy at our center. The patient inclusion criteria for our retrospective study were as follows: (1) thoracic superficial SCC (Tis and T1); (2) radical lymphadenectomy through a thoracotomy; (3) 12 or more removed lymph nodes ${ }^{11}$; (4) no chemotherapy or radiotherapy before surgery; and (5) specimens available for repeat review (Figure 1). A total of 189 patients met the inclusion 


\section{Abbreviations and Acronyms}

3-FL $=3$-field lymphadenectomy

$\mathrm{LNM}=$ lymph node metastasis

$\mathrm{SCC}=$ squamous cell carcinoma

SEC $=$ superficial esophageal cancer

criteria, with a mean patient age of $58 \pm 8$ years (range, 38-79), male-tofemale ratio of 3.2:1, and in-hospital mortality of $0.5 \%$ ( 1 of 189).

\section{Preoperative Workup}

According to the practice guidelines of the Fudan University Shanghai Cancer Center, the preoperative workup included a complete history, physical examination, endoscopy of the entire upper gastrointestinal tract, and histologic confirmation of the carcinoma. Computed tomography of the chest, ultrasonography or computed tomography of the abdomen, and the neck were performed to evaluate the resectability of the tumor and to exclude distant metastases.

\section{Surgical Procedure}

Surgery with curative intent involved resection of the primary cancer with negative microscopic resection margins and radical lymphadenectomy. The McKeown procedure, Ivor-Lewis procedure, and Sweet procedure were the 3 most common surgery types. Details of the resection techniques have been previously described. ${ }^{2}$ In brief, total mediastinal lymphadenectomy was routinely performed through a right-sided approach (McKeown procedure for upper esophageal tumors and Ivor-Lewis procedure for middle and lower tumors), and on the left using the Sweet procedure for lower esophageal tumors. Only the lymph nodes in the middle and lower mediastinum were removed because of the anatomic limitations. A gastric conduit was used for reconstruction in all patients. Cervical lymphadenectomy through a collar incision was included when nodal involvement was suggested by ultrasound or computed tomography.

\section{Lymph Node Classification}

Lymph node sites were classified according to the nomenclature and code number of the Japanese Society for Esophageal Disease. ${ }^{12}$ Cervical nodes included those in the cervical paraesophageal (no. 101) and supraclavicular regions (no. 104). The upper mediastinal nodes included the upper paraesophageal (no. 105) and paratracheal nodes (no.106r and 106tb). The middle mediastinal nodes included the subcarinal (no. 107), middle paraesophageal (no. 108), and bilateral hilar lymph nodes (no. 109). The lower mediastinal nodes included the lower paraesophageal (no. 110), posterior mediastinal lymph (no. 111), and diaphragmatic nodes (no. 112). The upper abdominal nodes included the nodes lateral to the paracardia, lesser curvature, greater curvature, left gastric, common hepatic, splenic, and celiac regions.

\section{Histopathologic Assessment}

The esophageal resection specimens were histopathologically assessed by experienced pathologists using a standardized protocol in which the site and size of the primary cancer, sample margins, and involvement of regional lymph nodes were recorded, in addition to the presence of lymphovascular invasion. Because subdivision of SEC is not routinely performed at our center, all SEC specimens were reviewed again by a pathologist (Y.K.), who was unaware of the previous pathologic reports, and subclassified into 6 layers according to the depth of invasion ${ }^{1}$ : epithelium (M1), superficial layer of the lamina propria mucosa (M2), deeper layer of the

\begin{tabular}{|c|c|}
\hline \multicolumn{2}{|c|}{$\begin{array}{c}\text { Patients under esophagectomy from } 2006 \\
\text { to } 2011(n=2069)\end{array}$} \\
\hline \multicolumn{2}{|c|}{ 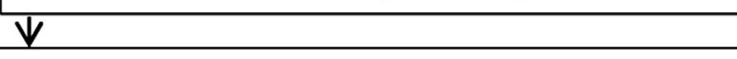 } \\
\hline \multicolumn{2}{|c|}{$\begin{array}{l}\text { Squamous cell carcinomas were included } \\
\qquad(n=1974)\end{array}$} \\
\hline \multirow{5}{*}{$\begin{array}{l}\text { Exclusion } \\
\text { criteria }\end{array}$} & Transhiatal esophagectomy $(n=21)$ \\
\hline & $\vee$ \\
\hline & $\begin{array}{l}\text { Chemotherapy and/or radiotherapy } \\
\text { prior to surgery }(n=35)\end{array}$ \\
\hline & $\downarrow$ \\
\hline & T2-4 tumors (1697) \\
\hline & $\boldsymbol{\psi}$ \\
\hline & Removed lymph nodes $<12(\mathrm{n}=25)$ \\
\hline & 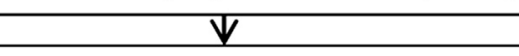 \\
\hline & Specimens unavailable $(n=7)$ \\
\hline
\end{tabular}

FIGURE 1. Patient disposition chart.

lamina propria mucosa (M3), superficial layer of the submucosa (SM1), middle layer of the submucosa (SM2), and deeper layer of the submucosa (SM3).

\section{Statistical Analysis}

Statistical analysis was performed using the Statistical Package for Social Sciences statistical software, version 16.0 (SPSS, Chicago, Ill). A nomogram and bootstrapping were done with $\mathrm{R}$, version 2.10.0. The standard chi-square test or Fisher's exact test was used for comparative analysis. Univariate and multivariate logistical regression analyses were performed to identify the factors associated with LNMs. The results were considered significant at $P<.05$.

\section{RESULTS}

All patients underwent curative R0 resection, of whom 23 underwent 3-field lymphadenectomy (3-FL), and 166 underwent 2-field lymphadenectomy. A total of 4252 lymph nodes were resected (average, $23 \pm 9$; range, 12-68). Of the 4252 lymph nodes resected, $107(2.5 \%)$ were positive in 49 of the 189 patients $(25.9 \%)$, including 2 of 47 patients $(4.3 \%)$ with mucosal and 47 of $142(33.1 \%)$ with submucosal cancer.

\section{Cancer Characteristics}

For analysis, the patients were divided into mucosal (M1, M2, M3) and submucosal (SM1, SM2 SM3) groups. The depth of invasion was M1 in 21, M2 in 9, M3 in 17, SM1 in 25, SM2 in 44, and SM3 in 73 patients. The baseline 
demographics, including age, gender, tumor location, and tumor length, were comparable between the 2 groups. No lymphovascular invasion was found in the mucosal tumors, whereas more poorly differentiated tumors were present in patients with submucosal tumors. Most patients $(78.8 \%)$ underwent radical 2-field lymphadenectomy through a right thoracotomy. The surgery types were similar between the 2 groups (Table 1). No LNMs occurred in patients with M1 or M2 cancer, but 2 of 17 patients $(11.8 \%)$ with M3 cancer had LNMs. The frequency of LNM was $24.0 \%, 20.5 \%$, and $43.8 \%$ for the SM1, SM2, and SM3 subgroups, respectively, with most submucosal cancers with LNM presenting with only 1 or 2 positive nodes (74.5\%, 35 of 47 cases).

\section{Risk Factors for LNMs in Submucosal Cancer}

Of the 142 patients with submucosal cancer, poor differentiation, lymphovascular invasion, and SM3 cancer

TABLE 1. Patient characteristics according to tumor invasion depth

\begin{tabular}{|c|c|c|c|}
\hline Characteristic & $\begin{array}{l}\text { Mucosal } \\
(\mathrm{n}=47)\end{array}$ & $\begin{array}{l}\text { Submucosal } \\
(\mathrm{n}=142)\end{array}$ & $\begin{array}{c}P \\
\text { value }\end{array}$ \\
\hline Gender & & & .387 \\
\hline Male & $38(80.9)$ & $106(74.6)$ & \\
\hline Female & $9(19.1)$ & $36(25.7)$ & \\
\hline Age $(y)$ & & & .830 \\
\hline$\leq 60$ & $26(55.3)$ & $76(53.5)$ & \\
\hline$>60$ & $21(44.7)$ & $66(46.5)$ & \\
\hline Tumor location & & & .357 \\
\hline Upper & $3(6.4)$ & $20(14.1)$ & \\
\hline Middle & $29(61.7)$ & $84(59.2)$ & \\
\hline Lower & $15(31.9)$ & $38(26.8)$ & \\
\hline Tumor length (cm) & & & .515 \\
\hline$\leq 2$ & $29(61.7)$ & $95(66.9)$ & \\
\hline$>2$ & $18(38.3)$ & $47(33.1)$ & \\
\hline Differentiation & & & $<.001$ \\
\hline Well & $26(55.3)$ & 15 (10.6) & \\
\hline Moderate & $21(44.7)$ & $90(63.4)$ & \\
\hline Poor & $0(0.0)$ & $37(26.1)$ & \\
\hline LNM & & & $<.001$ \\
\hline Negative & $45(95.7)$ & $95(66.9)$ & \\
\hline Positive & $2(4.3)$ & $47(33.1)$ & \\
\hline Lymphovascular invasion & & & .040 \\
\hline Yes & $0(0.0)$ & $12(8.5)$ & \\
\hline No & $47(100.0)$ & $130(91.5)$ & \\
\hline Procedure type & & & .260 \\
\hline Right thoracotomy (3-FL) & $3(6.4)$ & $20(14.1)$ & \\
\hline Right thoracotomy (2-FL) & 38 (80.9) & $111(78.2)$ & \\
\hline Left thoracotomy (2-FL) & $6(12.8)$ & $11(7.7)$ & \\
\hline \multicolumn{4}{|l|}{$\begin{array}{l}\text { Subclassification of tumor } \\
\text { invasion }\end{array}$} \\
\hline & M1: 21 (44.7) & SM1: 25 (17.6) & \\
\hline & M2: 9 (19.1) & SM2: 44 (31.0) & \\
\hline & M3: 17 (36.2) & SM3: 73 (51.4) & \\
\hline
\end{tabular}

Data presented as $\mathrm{n}(\%)$. LNM, Lymph node metastasis; 3-FL, 3-field lymphadenectomy; 2-FL, 2-field lymphadenectomy; $S M$, submucosa.
TABLE 2. Submucosal tumors: frequency of lymph node metastases according to tumor characteristics

\begin{tabular}{llc}
\hline \multicolumn{1}{c}{ Factor } & LNMs (\%) & P value \\
\hline Tumor location & & .400 \\
$\quad$ Upper & $20.0(4 / 20)$ & \\
Middle & $35.7(30 / 84)$ & \\
$\quad$ Lower & $34.2(13 / 38)$ & .415 \\
Tumor length (cm) & & \\
$\leq 1$ & $27.6(8 / 29)$ & \\
$1-\leq 2$ & $28.8(19 / 66)$ & \\
$2-\leq 3$ & $42.9(12 / 28)$ & \\
$>3$ & $42.1(8 / 19)$ & \\
Tumor differentiation & & \\
Well & $26.7(4 / 15)$ & \\
Moderate & $26.7(24 / 90)$ & \\
Poor & $51.4(19 / 37)$ & $.003 *$ \\
Lymphovascular invasion & & \\
No & $28.5(37 / 130)$ & \\
Yes & $83.3(10 / 12)$ & \\
Tumor invasion depth & & \\
SM1 & $24.0(6 / 25)$ & \\
SM2 & $20.5(9 / 44)$ & \\
SM3 & $43.8(32 / 73)$ & \\
\hline
\end{tabular}

$L N M s$, Lymph node metastases; $S M$, submucosa. *SM3 tumors compared with SM1 and SM2 tumors.

were associated with greater LNM occurrence. A tendency was seen for more LNM to occur as the tumor length increased, but no significant difference was observed (Table 2). Multivariate analysis identified SM3 cancer $(P=.006)$ and lymphovascular invasion $(P=.001)$ as independent LNM risk factors (Table 3). However, SM1 or SM2 cancers without lymphovascular invasion did not exclude the possibility of LNMs. The prediction model is presented as a nomogram that graphically shows the multivariate effects of the risk factors (Figure 2). The bootstrap-corrected c-index of the model was 0.74 .

\section{Pattern of LNMs}

For upper and middle submucosal cancer, LNM was common in the upper mediastinum and abdomen. For lower submucosal cancer, LNM was common in the lower mediastinum and abdomen (Figure 3). For submucosal cancers, paratracheal lymph nodes were the most frequently involved $(12.0 \%)$, followed by the lesser curvature $(10.6 \%)$, middle paraesophageal $(5.6 \%)$, and paracardial nodes $(5.6 \%)$ (Figure 4$)$.

"Skip" metastases, referring to a patient with cervical and/or abdominal involvement but no mediastinal metastasis, occurred in 20 of 49 patients $(40.8 \%)$. Of those with skip metastases, 2 had M3 tumors. Positive LNMs involving 2 regions were seen in $16.7 \%$ of SM1, $30 \%$ of SM2, and $16.1 \%$ of SM3 tumors. Cervical metastases were identified in only 1 of 23 patients $(0.4 \%)$ with 3 -FL (Table 4). 
TABLE 3. Submucosal tumors: logistic analysis of risk factors for lymph node metastases

\begin{tabular}{|c|c|c|c|c|c|c|}
\hline \multirow[b]{2}{*}{ Variable } & \multicolumn{3}{|c|}{ Univariate analysis } & \multicolumn{3}{|c|}{ Multivariate analysis } \\
\hline & OR & $\mathbf{9 5} \%$ CI & $P$ value & OR & $95 \%$ CI & $P$ value \\
\hline Tumor depth & & $1.347-5.863$ & .006 & & $1.361-6.550$ & .006 \\
\hline SM1/SM2 & 1 & & & 1 & & \\
\hline SM3 & 2.810 & & & 2.855 & & \\
\hline Lymphovascular invasion & & $2.672-60.122$ & .002 & & $2.726-68.008$ & .001 \\
\hline No & 1 & & & 1 & & \\
\hline Yes & 12.568 & & & 13.615 & & \\
\hline Tumor length $(\mathrm{cm})$ & & $0.899-3.872$ & .094 & - & - & - \\
\hline$\leq 2$ & 1 & & & & & \\
\hline$>2$ & 1.866 & & & & & \\
\hline Differentiation & & $1.336-6.309$ & .007 & - & - & - \\
\hline Well and moderate & 1 & & & & & \\
\hline Poor & 2.903 & & & & & \\
\hline
\end{tabular}

$O R$, Odds ratio; $C I$, confidence interval; $S M$, submucosa

\section{DISCUSSION}

Esophageal cancer is the eighth most common cancer and sixth most common cause of cancer-related death in China. ${ }^{13}$ At diagnosis, most esophageal cancers are at a locally advanced stage. However, because of widespread endoscopic screening and advances in diagnostic modalities, such as endoscopic mucosal resection and endoscopic submucosal dissection, the incidence of SEC in China is increasing. Thus, less invasive procedures without lymphadenectomy, including endoscopic therapy, have been increasingly performed. In contrast to the West, where adenocarcinoma is the major pathologic type, SCC is the predominate type in China. Because the lymph node status is one of the most important factors in predicting the prognosis for patients with esophageal SCC, it is crucial to know the risk of LNMs for the management of SEC. Therefore, in the present study, we aimed to accurately document the incidence of LNMs and study the factors associated with LNMs in patients with superficial SCC.

Our results also showed that LNM was rare when the cancer was confined to the mucosa $(4.3 \%$, a rate similar to previous reports $\left.{ }^{7,14}\right)$. No LNM was observed in patients with M1 or M2 tumors; therefore, endoscopic resection can be indicated for such patients. However, in patients with M3 tumors, LNMs were found in 2 of 17 patients, 1 of whom had 2 positive lymph nodes in the mediastinum and 1 of who had 3 in the abdomen. Both tumors were moderately differentiated and without lymphovascular invasion; the reason for the LNM was perhaps related to the greater tumor size. No LNMs were found in patients
Points

Depth of Tumor Invasion

Lymphovascular Invasion

Tumor Differentiation

Tumor Length

Total Points

Predicted Risk of Lymph Node Metastases

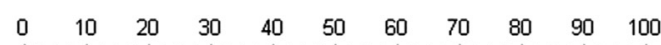

SM1
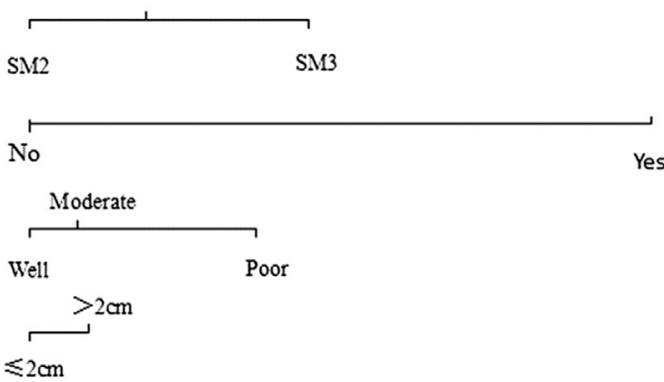

$\begin{array}{llllllllllll}0 & 20 & 40 & 60 & 80 & 100 & 120 & 140 & 160 & 180 & 200 & 220\end{array}$

FIGURE 2. Nomogram predicting risk of lymph node metastases in submucosal cancers. Nomogram instructions: to obtain the nomogram-predicted risk of lymph node metastases, locate the patient values on each axis. Draw a vertical line to the points axis to determine how many points are attributable to each variable. Sum the points for all the variables. Locate the sum on the total points line to assess the patient's predicted risk of lymph node metastases. SM, Submucosa. 


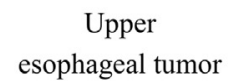

$(n=20)$

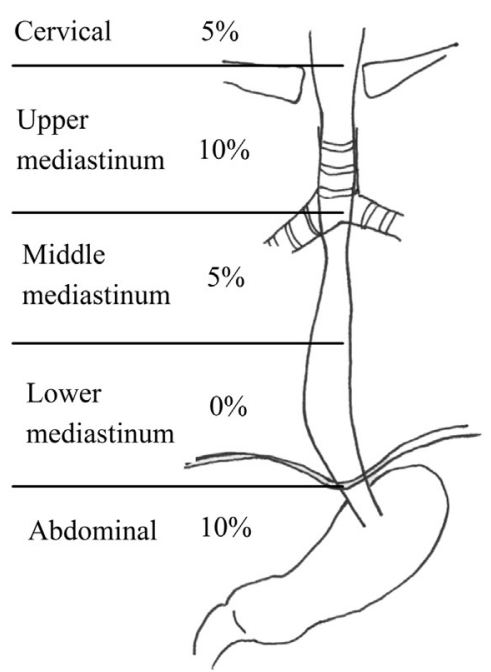

Middle

esophageal tumor $(\mathrm{n}=84)$

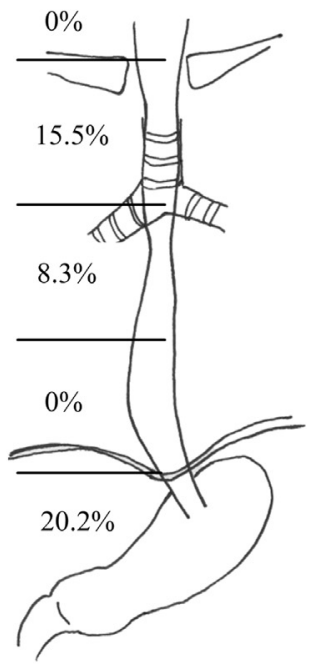

Lower

esophageal tumor $(\mathrm{n}=38)$

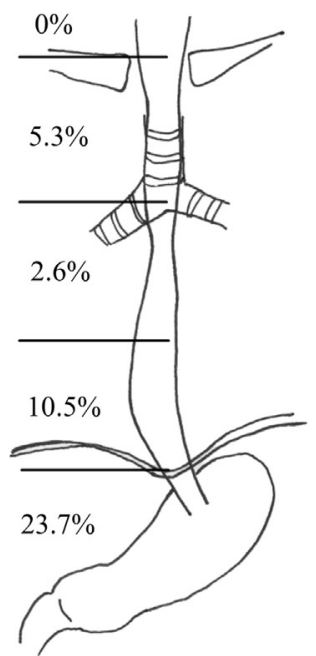

FIGURE 3. Frequency of lymph node metastases according to tumor location in submucosal tumors.

with M3 tumors less than $2 \mathrm{~cm}$, and the rate was $28.6 \%$ ( 2 of 7 ) when the tumors were larger than $2 \mathrm{~cm}$. Thus, our results suggest that endoscopic mucosal resection can be performed for most M3 tumors but that extended 2-field lymphadenectomy should be considered when the lesion is larger than $2 \mathrm{~cm}$.

Compared with the lower LNM risk for an intramucosal lesion, the incidence of LNM increased dramatically when the cancer had invaded into the submucosa. One major controversy is whether endoscopic therapy is suitable for submucosal lesions. Some have considered that selected SEC tumors less than $2 \mathrm{~cm}$ in diameter, with no lymphatic vessel invasion and well to moderate differentiation can be treated endoscopically. ${ }^{7,8,15}$ In our series, only the depth of tumor invasion and lymphovascular invasion were identified as independent LNM risk factors. However, the

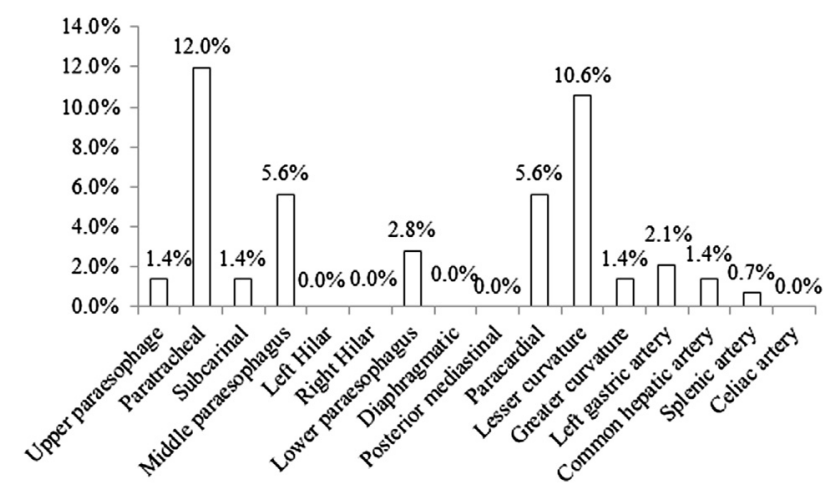

FIGURE 4. Frequency of lymph node metastases according to anatomic site in submucosal tumors. absence of any of these factors did not exclude the possibility of LNM. We also found tumor size, a risk factor in previous reports, ${ }^{7,16,17}$ to be of less importance in the assessment of LNM for submucosal tumors. No significant difference was found between the incidence of LNM between smaller tumors $(\leq 2 \mathrm{~cm})$ and larger tumors $(>2 \mathrm{~cm})$. The comparable incidence of early and skip metastasis along the abundant lymphatic channels in the mucosa and submucosa between the smaller and larger tumors is a possible reason. Consistent with a published report referring to the issue, ${ }^{18}$ our data also indicated that patients should not be recommended for endoscopic treatment when the cancer has invaded the submucosa.

The optimal extent of lymph node resection for submucosal cancer remains unclear owing to the complex pattern of LNMs and lack of prospective studies., ${ }^{3,4}$ To date, few studies have focused on the pattern of LNM in superficial

TABLE 4. Region of lymph node metastases according to tumor invasion depth

\begin{tabular}{lcccc}
\hline & $\begin{array}{c}\text { M3 } \\
\text { (2 cases) }\end{array}$ & $\begin{array}{c}\text { SM1 } \\
\text { (6 cases) }\end{array}$ & $\begin{array}{c}\text { SM2 } \\
\text { (10 cases) }\end{array}$ & $\begin{array}{c}\text { SM3 } \\
\text { (31 cases) }\end{array}$ \\
\hline One region & & & & \\
$\quad$ Neck & & & & 1 \\
$\quad$ Mediastinum & 1 & 3 & 2 & 14 \\
$\quad$ Abdomen & 1 & 2 & 5 & 11 \\
Two regions & & & & \\
$\quad$ Mediastinum plus neck & & 0 & 0 & 0 \\
Abdomen plus neck & & 0 & 0 & 0 \\
$\quad$ Mediastinum plus abdomen & & 1 & 3 & 5 \\
\hline
\end{tabular}

SM, Submucosa. 
esophageal cancer. The present study is one of the largest series reported to determine the pattern of LNMs in patients with superficial SCC. Most of our patients with LNMs had only 1 or 2 positive lymph nodes, ranging from the neck to the upper abdomen. One notable finding was that the LNMs mostly occurred in the upper mediastinum, paraesophageal, and perigastric regions. Paratracheal lymph nodes were the most frequently involved, followed by paraesophageal and perigastric nodes; thus, lymph nodes in these areas should be carefully removed. A second finding was that skip metastases were common $(40.8 \%)$. Cervical metastases, however, were rare. Of the 20 patients with submucosal cancer who underwent 3-FL, only 1 patient (SM3 cancer, upper third tumor location) presented with cervical involvement. Therefore, 2-field radical lymphadenectomy would seem to be sufficient for T1b cancer, although lymph node resection in the upper mediastinum remains necessary.

One limitation of the present study was that it was retrospective. Also, nodal micrometastasis found on immunohistochemistry is common. It has been reported that almost $27 \%$ of patients with superficial SCC with routine histologically node-negative esophageal cancer (pN0) will, in fact, be found to harbor nodal micrometastases on immunohistochemistry, ${ }^{19}$ although we did not examine this. In addition, the extent of lymphadenectomy was affected by the surgical procedure type and surgeon preference. Thus, lymph nodes in the upper mediastinum were always omitted when a left thoracotomy was used, cervical lymphadenectomy was not systematically undertaken, and the proportion of 3-FL was low. However, most SEC studies have covered more than a 10 -year period, and our database originated from a single, high-volume institute within a short period. Thus, our data were more uniform. Because endoscopic therapy has been performed increasingly since 2011 at our center, the long-term survival of patents who underwent endoscopic and surgical treatment will be reported in future studies.

In conclusion, our results indicate that patients with cancer limited to the lamina propria mucosa have a low risk of LNMs. Endoscopic treatment is an appropriate option for such mucosal cancers, although attention is needed when the tumor is larger than $2 \mathrm{~cm}$. Because of the high incidence of LNMs, patients with submucosal cancer, however, should not be recommended for endoscopic treatment. According to the pattern of lymphatic spread in our study, 2-field radical lymphadenectomy with careful lymph node resection in the upper mediastinum should be performed for submucosal SCC.
We thank Huixun Jia (Department of Biostatistics, Fudan University Shanghai Cancer Center) for her contribution to the statistical analysis in our study.

\section{References}

1. Japanese Society for Esophageal Disease Guidelines for the clinical and pathological studies on carcinoma of the esophagus. Jpn J Surg. 1976;6: 69-78.

2. Fujita H, Sueyoshi S, Tanaka T, Fujii T, Toh U, Mine T, et al. Optimal lymphadenectomy for squamous cell carcinoma in the thoracic esophagus: comparing the short- and long-term outcome among the four types of lymphadenectomy. World J Surg. 2003;27:571-9.

3. Grotenhuis BA, van Heijl M, Zehetner J, Moons J, Wijnhoven BP, van Berge Henegouwen MI, et al. Surgical management of submucosal esophageal cancer: extended or regional lymphadenectomy? Ann Surg. 2010;252:823-30.

4. Tachibana M, Hirahara N, Kinugasa S, Yoshimura H. Clinicopathologic features of superficial esophageal cancer: results of consecutive 100 patients. Ann Surg Oncol. 2008;15:104-16.

5. Ishihara R, Iishi H, Uedo NT, Takeuchi Y, Yamamoto S, Yamada T, et al. Comparison of EMR and endoscopic submucosal dissection for en bloc resection of early esophageal cancers in Japan. Gastrointest Endosc. 2008;68:1066-72.

6. Shi Q, Zhong YS, Yao LQ, Zhou PH, Xu MD, Wang P. Endoscopic submucosal dissection for treatment of esophageal submucosal cancers originating from the muscularis propria layer. Gastrointest Endosc. 2011;74:1194-200.

7. Endo M, Yoshino K, Kawano T, Nagai K, Inoue H. Clinicopathologic analysis of lymph node metastasis in surgically resected superficial cancer of the thoracic esophagus. Esophagus. 2000;13:125-9.

8. Kim DU, Lee JH, Min BH, Shim SG, Chang DK, Kim YH, et al. Risk factors of lymph node metastasis in T1 esophageal squamous cell carcinoma. J Gastroenterol Hepatol. 2008;23:619-25.

9. Eguchi T, Nakanishi Y, Shimoda T, Iwasaki M, Igaki H, Tachimori Y, et al. Histopathological criteria for additional treatment after endoscopic mucosal resection for esophageal cancer: analysis of 464 surgically resected cases. Mod Pathol. 2006;19:475-80.

10. Igaki H, Kato H, Tachimori Y, Nakanishi Y. Cervical lymph node metastasis in patients with submucosal carcinoma of the thoracic esophagus. J Surg Oncol. 2000;75:37-41.

11. Dutkowski P, Hommel G, Böttger T, Schlick T, Junginger T. How many lymph nodes are needed for an accurate $\mathrm{pN}$ classification in esophageal cancer? Evidence for a new threshold value. Hepatogastroenterology. 2002;49:176-80.

12. Natsugoe S, Matsumoto M, Okumura H, Nakashima S, Higashi H, Uenosono Y, et al. Initial metastatic, including micrometastatic, sites of lymph nodes in esophageal squamous cell carcinoma. J Surg Oncol. 2005;89:6-11.

13. Parkin DM, Bray F, Ferlay J, Pisani P. Global cancer statistics, 2002. CA Cancer J Clin. 2005;55:74-108.

14. Shimada H, Nabeya Y, Matsubara H, Okazumi S, Shiratori T, Shimizu T, et al Prediction of lymph node status in patients with superficial esophageal carcinoma: analysis of 160 surgically resected cancers. Am J Surg. 2006;191:250-4.

15. Higuchi K, Tanabe S, Koizumi W, Sasaki T, Nakatani K, Saigenji K, et al. Expansion of the indications for endoscopic mucosal resection in patients with superficial esophageal carcinoma. Endoscopy. 2007;39:36-40.

16. Bolton WD, Hofstetter WL, Francis AM, Correa AM, Ajani JA, Bhutani MS, et al. Impact of cancer length on long-term survival of pT1 esophageal adenocarcinoma. J Thorac Cardiovasc Surg. 2009;138:831-6.

17. Leers JM, DeMeester SR, Oezcelik A, Klipfel N, Ayazi S, Abate E, et al The prevalence of lymph node metastases in patients with $\mathrm{T} 1$ esophageal adenocarcinoma: a retrospective review of esophagectomy specimens. Ann Surg. 2011;253:271-8.

18. Ancona E, Rampado S, Cassaro M, Battaglia G, Ruol A, Castoro C, et al Prediction of lymph node status in superficial esophageal carcinoma. Ann Surg Oncol. 2008;15:3278-88.

19. Prenzel KL, Hölscher AH, Drebber U, Agavonova M, Gutschow CA Bollschweiler E. Prognostic impact of nodal micrometastasis in early esophageal cancer. Eur J Surg Oncol. 2012;38:314-8. 